\title{
PRELIMINARY ANALYSIS OF A HUBBLE FOS SPECTRUM OF VW HYI IN QUIESCENCE: A DAZQ WHITE DWARF AND ACCRETION BELT/RING
}

\author{
E. M. SION ${ }^{1}$, M. HUANG ${ }^{1}$, F. H. CHENG ${ }^{1}$, I. HUBENY ${ }^{2}$ \\ P. SZKODY ${ }^{3}$ \\ 1. Villanova University, Villanova, PA 19085, USA \\ 2. Laboratory of Astronomy \& Solar Physics, \\ Code 681, NASA/GSFC, Greenbelt, MD 20771, USA \\ 3. Dept. of Astronomy, FM-20, \\ Univ. of Washington, Seattle, WA 98195, USA
}

\begin{abstract}
We present a preliminary analysis of the UV spectrum of the dwarf nova VW Hyi during early quiescence, obtained with the Faint Object Spectrograph (FOS) on the Hubble Space Telescope (HST). The data were obtained approximately one day after the end of a normal outburst.
\end{abstract}

\section{HST FOS spectrum and line measurements}

We obtained a Hubble Space Telescope FOS spectrum of VW Hyi on 1995 January 28, 19:33:01 (UT time), approximately one day after the end of a normal outburst. The spectrum is dominated by a very broad Ly $\alpha$ profile and a rich metallic absorption line spectrum similar to our earlier FOS spectrum obtained ten days after superoutburst (see Sion et al. 1995; Huang et al. 1996). There is a moderately strong, broad C IV emission line associated with the Keplerian broadening of an accretion disk. A list of identified lines with equivalent widths (EW) and full widths at half-maximum (FWHM) are presented in Table 1.

\section{Preliminary synthetic spectral analysis results}

There are important differences between this new FOS spectrum and the one analyzed in Sion et al. (1995). Our new spectrum reveals that the Ly $\alpha$ absorption line is measurably narrower, the continuum hump be- 
TABLE 1. Absorption lines in the FOS spectrum of VW Hyi

\begin{tabular}{llll}
\hline Line & Wavelength $(\AA)$ & EW $(\AA)$ & FWHM $(\AA)$ \\
\hline C III $_{\text {Si II }}$ & 1176.7 & 3.153 & 5.684 \\
& 1261.2 & 1.685 & 3.161 \\
C I $_{\text {O I + Si III + Si II }} 1265.2$ & 2.083 & 3.929 \\
Si III & 1278.55 & 3.448 & 9.406 \\
C II $_{\text {Si IV }}$ & 1310.59 & 9.079 & 18.78 \\
Si II & 1335.78 & 2.381 & 5.872 \\
& 1395.28 & 2.262 & 4.094 \\
C I $_{\text {C IV }}^{1527.52}$ & 1.721 & 7.923 \\
& 1533.77 & 1.712 & 5.463 \\
& 1562.14 & 1.929 & 9.868 \\
\hline
\end{tabular}

* Emission line

tween $1400 \AA$ and $1550 \AA$ is not as pronounced, and the overall flux level is slightly higher. We interpret these differences as indicative of a higher overall surface temperature for the white dwarf one day after a normal outburst than its overall surface temperature ten days after superoutburst. Our best synthetic spectral fit to the data is a composite two-temperature model consisting of (i) a DAZQ white dwarf with $T_{\text {eff }}=22000 \mathrm{~K}, \log g=8$, and abundances $\mathrm{C} / \mathrm{C}_{\odot}=0.5, \mathrm{~N} / \mathrm{N}_{\odot}=5.0, \mathrm{O} / \mathrm{O}_{\odot}=0.02, \mathrm{Si} / \mathrm{Si} \odot=0.2$, $\mathrm{Fe} / \mathrm{Fe}_{\odot}=0.5$ with all other metals being 0.3 solar and (ii) a rapidly spinning accretion belt (or possibly inner disk ring) with $V_{\text {rot }}=3350 \mathrm{~km} \mathrm{~s}^{-1}$, $T_{\text {belt }}=28000 \mathrm{~K}, \log g=6.0$, overabundance of $\mathrm{C}$ and $\mathrm{Si}$ by 20 times and 15 times solar, respectively, and a fractional belt area of approximately $6 \%$. A detailed comparison of this second FOS spectrum, taken one day post-outburst, with the first FOS spectrum obtained ten days post superoutburst, is now in progress.

Acknowledgement. This work is supported by NASA grants GO-3836.0191A, GO-6084.01-95A and NAGW-5726 to Villanova University.

\section{References}

Huang, M., Sion, E. M., Hubeny, I., et al., 1996, these proceedings, p247

Sion, E.M., Szkody, P., Cheng, F.H., Huang, M., 1995, Ap. J., 444, L97 